\title{
Cyclotron resonance for two-dimensional electrons on thin helium films
}

\author{
J. Klier, A. Würl, and P. Leiderer \\ Fakultät für Physik, Universität Konstanz, D-78457 Konstanz, Germany \\ G. Mistura \\ Universita di Padova, 35131 Padova, Italy \\ V. Shikin \\ ISSP, 142432 Chernogolovka, Moscow District, Russia
}

(Received 19 January 2002; published 10 April 2002)

\begin{abstract}
We present a systematic investigation of the microwave absorption for two-dimensional electron layers on thin helium films and in the presence of a cyclotron resonance (CR) magnetic field. To explain the measured data, a recently proposed two-fraction structure of the electron system is used and here described in detail. Hereby the problem of substrate roughness, usually always present for electrons on thin helium films, is taken into account and it turns out to be an important parameter. Within this model the general structure of the microwave absorption becomes understandable and the fraction of localized and free electrons can be precisely determined. The details of the observed asymmetry and shift of the CR line shape are discussed.
\end{abstract}

DOI: 10.1103/PhysRevB.65.165428

PACS number(s): $67.70 .+\mathrm{n}, 72.10 .-\mathrm{d}, 72.60 .+\mathrm{g}, 73.50 .-\mathrm{h}$

\section{INTRODUCTION}

A two-dimensional (2D) sheet of electrons on thin helium films forms traditionally an interesting field for studying low-dimensional systems. So there is, e.g., the "dimple" formation, ${ }^{1-4}$ the high level of stability (with respect to the bulk situation), ${ }^{5-8}$ the dipole-dipole crystallization, ${ }^{9,10}$ the layering effect in the electron mobility, ${ }^{11,12}$ and so on. All these phenomena are developed under the assumption that the solid substrate is flat. However, in reality solid surfaces are not perfect and the typical level of roughness is usually not small (the roughness amplitude is comparable to the helium film thickness). Under these conditions the question arises of how the 2D electron system on a thin helium film "feels" the existing random roughness of the substrate. A preliminary answer to this question is presented in Ref. 13. Using quite general assumptions 2D electron layers on thin helium films are represented as a two-fraction system which leads to various consequences of the understanding of these electron layers. ${ }^{13}$ In this paper the two-fraction scenario is systematically developed for the cyclotron resonance (CR) problem. We explain, how the free electron motion and localization phenomena can coexist in the presence of randomly rough solid substrates under CR conditions.

\section{CORRUGATED HELIUM FILM WITHOUT 2D ELECTRONS}

To describe the behavior of a helium film adsorbed on a corrugated substrate we first consider the neutral situation, i.e., without electrons on the helium surface.

(1) Let us assume the periodic substrate profile $\delta(x)$ as

$$
\delta(x)=h+ \begin{cases}\delta_{0}, & 0 \leqslant x \leqslant a, \\ -\delta_{0}, & a<x<(a+b) .\end{cases}
$$

Here $(a+b)$ is the corrugated structure period and $h$ $\left(>2 \delta_{0}\right)$ is the distance between the average structure of the substrate and the bulk liquid helium surface.

For simplification we only discuss the limiting case

$$
b^{2}<\frac{\sigma_{l v}}{\rho g},
$$

i.e., the width of the structures is assumed to be small compared to the capillary length of the liquid helium. $\sigma_{l v}$ is the surface tension, $\rho$ the bulk helium density, and $g$ the acceleration due to gravity. The properties of a neutral helium film $d(x)$ on a rough substrate can be extracted from

$$
\sigma_{l v} \frac{d^{\prime \prime}(x)}{\left[1+\left(d^{\prime}\right)^{2}\right]^{3 / 2}}-\rho g d(x)+\frac{C_{3}}{d^{3}(x)}=\rho g h,
$$

where $C_{3}$ is the van der Waals constant of the helium-solid substrate boundary.

In the case of a uniform helium film, when $\delta_{0} \rightarrow 0$, Eq. (3) is reduced to the conventional definition of the helium film thickness $d$ (for simplicity the retardation effect for very thick films, ${ }^{14}$ where $d$ depends with the fourth power on $h$, is not considered here)

$$
d^{3}=\frac{C_{3}}{\rho g h} .
$$

In the presence of a regular corrugation $\delta(x) \neq 0$ the question arises about the properties of the nonuniform thickness $d(x)$. One can show that then

$$
d(x)= \begin{cases}d_{\text {top }}, & 0 \leqslant x \leqslant a, \\ R+d_{\text {min }}-\sqrt{R^{2}-(x-c)^{2}}, & a<x<(a+b),\end{cases}
$$

where 


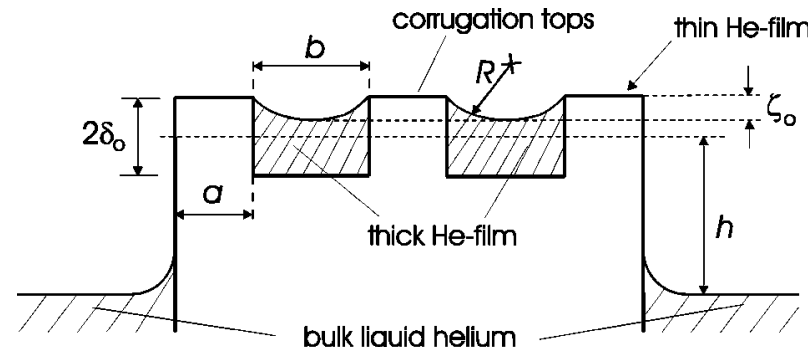

FIG. 1. Shown is a schematical sketch of a corrugated surface where, due to capillary condensation, a suspended thick liquid helium film exists. The symbols are explained in the text.

$$
\begin{gathered}
d_{\text {top }}^{3}=\frac{C_{3}}{\rho g\left(h+\delta_{0}\right)}, \quad \frac{2 \sigma_{l v}}{R} \simeq \rho g h, \quad c=a+\frac{b}{2}, \\
d_{\text {min }}=2 \delta_{0}-\zeta_{0},
\end{gathered}
$$

and

$$
\zeta_{0}=R-\sqrt{R^{2}-b^{2} / 4}
$$

$R$ is the radius of curvature of the capillary condensed thick helium film, see Fig. 1. The structure of helium films of this kind has been visually investigated in Ref. 15 .

Using definition (5) we can formulate the conditions for a weak and strong helium film corrugation; the corrugation is weak, if

$$
\delta_{0}^{2} \ll d^{2} \quad \text { or } \quad \zeta_{0} \ll d .
$$

In the opposite limiting case we have the strong corrugation situation.

The first case of condition (8) is evident. It corresponds to the perturbative coexistence between the helium film and the corrugation of the solid substrate with zero approximation for $d$ from Eq. (4). The details and behavior of $d(x)$ versus $\delta(x)$ in this limit are investigated, e.g., in Ref. 16.

More interesting for the roughness problem below is the second case, i.e., when the helium film profile does not follow the corrugation $\delta(x)$ of the solid substrate. In the limit $R \gg b$, when $\zeta_{0} \rightarrow 0$, then $d_{\min } \rightarrow 2 \delta_{0}$. Therefore in this limit the definition of $d$ (4) correlates only with $d_{\text {top }}(6)$. The behavior of $d(x)$ between the tops is controlled mainly by the Laplace pressure with the essential screening of the profile $\delta(x)$ below the helium surface. Such a behavior has interesting consequences for a rough substrate, because a small amount of the strong random deviations in the distribution $\delta(x)$ together with the effect of the Laplace screening between these tops can control all the behavior of the helium film $d(x)$ along the rough substrate.

(2) Roughness is a property of most solid substrates. Usually it is assumed that a one-dimensional random roughness behavior $\delta(x)$ can be described by a Gaussian distribution of the amplitudes

$$
G(\delta)=\frac{1}{\left(2 \pi \Delta^{2}\right)^{1 / 2}} \exp \left(-\frac{\delta^{2}}{2 \Delta^{2}}\right)
$$

where $\Delta^{2}=\left\langle\delta^{2}\right\rangle$ is the mean-square roughness amplitude in vertical direction. if

In terms of Eq. (9) the influence of the roughness is weak,

$$
\Delta^{2} \ll d^{2} \text {. }
$$

But, after the discussion of Eqs. (1)-(10) it becomes clear that the limitation (10) is not enough to avoid the problem of roughness in the behavior of $d(x)$. Even a small amount of high roughness tops with $\delta^{2} \gg \Delta^{2}$ can be important for the details of the $d(x)$ dependence.

To introduce the Laplace screening effect in the calculation of $d(x)$ we need some additional basic definitions. One of them is the general expression for the average density of the high enough tops $n_{\delta}$ above the fixed level $\delta>0$ (Ref. 17)

$$
n_{\delta}=\frac{1}{2 \pi} \sqrt{-Z^{\prime \prime}(0)} \exp \left(-\frac{\delta^{2}}{2 \Delta^{2}}\right)
$$

where

$$
Z^{\prime \prime}(0)=-\left(\frac{2 \pi}{\Delta}\right)^{2} \int_{0}^{\infty} w S(w) d w
$$

$S(w)$ is the spectral density function for the roughness distribution, Eq. (9), with the correlation function

$$
\left\langle\delta(x) \delta\left(x-x^{\prime}\right)\right\rangle=\Delta^{2} \exp \left(-\frac{x^{\prime 2}}{2 \eta^{2}}\right) .
$$

Here $\left\langle\eta^{2}\right\rangle$ is the correlation length in horizontal direction.

After calculations, see Ref. 17, one gets from Eqs. (11) and (12)

$$
n_{\delta}=\frac{1}{s \eta} \exp \left(-\frac{\delta^{2}}{2 \Delta^{2}}\right), \quad s^{2}=2 \pi, \quad \eta=\sqrt{\left\langle\eta^{2}\right\rangle} .
$$

Here $\Delta^{2}$ is from Eq. (9) and $\left\langle\eta^{2}\right\rangle$ is from Eq. (12).

To couple the arbitrary level $\delta>0$ [Eq. (13)] with the Laplace radius $R$, we assume that for the Laplace length $b$ we have

$$
b=n_{\delta}^{-1} .
$$

Figure 2 explains the definition of the geometric size $b$ and helps to understand the correlation between $b, R$, and $\zeta_{0}$, i.e., $\zeta_{0}=R-\sqrt{R^{2}-b^{2} / 4}$, see Eq. (7) and also Fig. 1. In addition, we require

$$
\frac{d b}{d \zeta_{0}}=\frac{d n_{\delta}^{-1}}{d \delta}
$$

This condition couples the characteristics of $R$ with the "speed" of the change of $n_{\delta}$ versus $\delta$.

The four definitions (7) and (13)-(15), are sufficient to express $\delta, b, \zeta_{0}$, and $n_{\delta}$ versus $R, \eta$, and $\Delta$. All these definitions are labeled by the index " $a$ " to indicate the connection of the density of active tops $n_{a}$ and the position of the active level $\delta_{a}$ to the problem of a helium film on a rough substrate with 


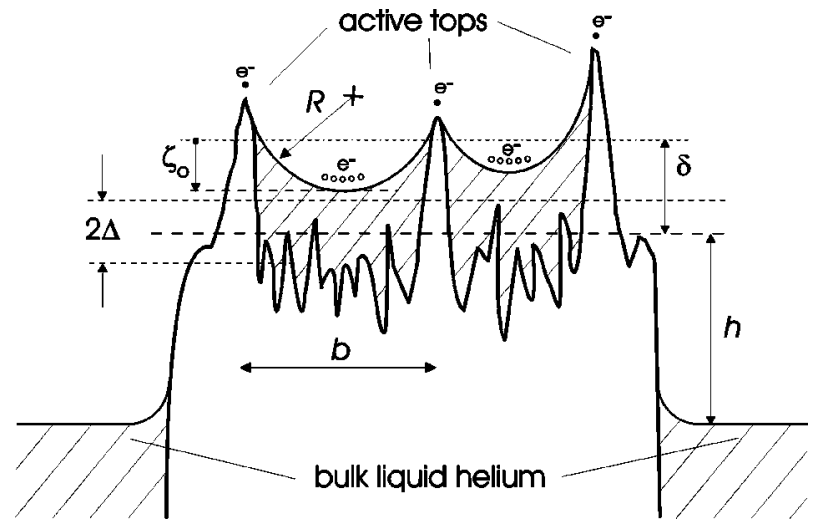

FIG. 2. Shown is a schematical cross section through a typical randomly rough substrate. Above the active tops only a thin van der Waals helium film exists with the localized electrons $(\mathbf{O})$ above the tops. In between the active tops a suspended thick helium film is formed with the quasifree electrons $(\bigcirc)$ above. The symbols are explained in the text.

$$
R=\frac{b_{a}}{2} \sqrt{1+\frac{b_{a}^{2}}{2 \Delta^{2}} \ln \frac{b_{a}}{s \eta}}
$$

and

$$
\frac{\delta_{a}^{2}}{2 \Delta^{2}}=\ln \frac{b_{a}}{s \eta}, \quad n_{a}^{-1}=b_{a}, \quad s \eta n_{a} \ll 1 .
$$

The screening effect (i.e., decreasing of $n_{a}$ versus $R$ ) is most pronounced if $R \gg \Delta$. In this case, and in the reasonable interval of " $h$ ", we have $b_{a}^{2} \gg \Delta^{2}$. Under these conditions we get

$$
R \simeq \frac{b_{a}}{2 \sqrt{2}}\left(\frac{b_{a}}{\Delta}\right) \ln ^{1 / 2} \frac{b_{a}}{s \eta} .
$$

So for the situation of the one-dimensional roughness one gets $b_{a} \approx R^{1 / 2}$.

In the two-dimensional case we have (if fluctuations are independent)

$$
n_{a}^{-1} \approx b_{a}^{2}
$$

In addition to $n_{a}$ it is reasonable to use $n_{a}^{T}$ as density of active tops. The reason is that all active tops above the level $\delta_{a}$ are sensitive to the pressing electric field $E_{\perp}$. Under these conditions the highest tops are unstable, and all local electrons will be localized in a narrow interval of $\delta<\delta_{a}$ with density $n_{a}^{T}$ such that

$$
n_{a}^{T} \simeq-\frac{T}{e E_{\perp}} \frac{d n_{a}}{d \delta}=n_{a} \frac{\delta_{a}}{\Delta^{2}} \frac{T}{e E_{\perp}} .
$$

The definition of $n_{a}^{T}$ (19) has practically the same $R$ dependence as $n_{a}$ (because $\delta_{a}$ is a "weak" function of $R$ ). By summarizing we can see that (a) the helium film above a rough substrate causes a finite density of active tops $n_{a}$, see Eqs. (18) or (19), even under condition (10); (b) $n_{a}$ of these tops is a continuous function of $h\left(n_{a} \rightarrow 0\right.$ if $\left.h \rightarrow 0\right)$, and it can be comparable with the total number of the $2 \mathrm{D}$ electrons $n_{s}$ (see Sec. III); (c) the helium film thickness on the tops is practically not sensitive to the distribution of $\delta$ with $\delta$ $>\delta_{a}$. Indeed, if $\Delta^{2} \ll h^{2}$ (such a requirement is fulfilled with high accuracy), the local helium film thickness, $d_{a}$, above the active roughness tops can be estimated from

$$
\frac{C_{3}}{d_{a}^{3}} \simeq \rho g\left(h+d_{a}\right)+\sigma_{l v}\left[d_{a}^{\prime \prime} d_{a}^{\prime \prime}\right]^{1 / 2} .
$$

If $\Delta^{2} \ll h^{2}$ and the local derivatives $d_{a}^{\prime \prime}$ above the active tops are less or comparable to $\delta^{\prime \prime}$, then

$$
d_{a}^{\prime \prime} d_{a}^{\prime \prime} \leqslant\left\langle d^{\prime \prime} d^{\prime \prime}\right\rangle \leqslant\left\langle\delta^{\prime \prime} \delta^{\prime \prime}\right\rangle, \quad\left\langle\delta^{\prime \prime} \delta^{\prime \prime}\right\rangle=\frac{3 \Delta^{2}}{\eta^{4}} .
$$

In this case definition (20) is reduced to

$$
\frac{C_{3}}{d_{a}^{3}} \simeq \rho g h+\sigma_{l v}\left\langle\delta^{\prime \prime} \delta^{\prime \prime}\right\rangle^{1 / 2},
$$

which is not sensitive to $\delta_{a}$. The definition for $\left\langle\delta^{\prime \prime} \delta^{\prime \prime}\right\rangle$ in Eq. (21) follows from the combination of Eq. (9) and the definition of the correlation function, Eq. (12), see Ref. 17.

\section{2D ELECTRONS ON A ROUGH HELIUM FILM, TWO-FRACTION STATISTICS}

In the presence of a substrate with active tops the 2Delectron system (2DES) on a thin helium film above this substrate is separated in two fractions. One fraction of the electron density $n_{e}$ corresponds to free electron motion along the helium surface. In Fig. 2 these electrons form the electron puddle between the active tops. The second fraction $n_{l}$ represents the density of electrons localized to potential wells of the roughness of the solid substrate. These electrons are localized in the vicinity of the tops above the helium film. It is evident that

$$
n_{e}+n_{l}=n_{s},
$$

where $n_{s}$ is the total 2D electron density, which is typically fixed. But the relationship between these fractions is flexible. This follows from the behavior of the equilibrium chemical potential $\mu_{0}$. To define $\mu_{0}$ we follow the procedure used for semiconductors, ${ }^{18}$ i.e.

$$
\begin{gathered}
n_{l}=\frac{n_{a}}{\exp \left[\left(V_{a}-\mu_{0}\right) / T\right]+1}, \quad V_{a}<0, \\
n_{e}=\frac{n_{0}^{e} \exp \left(T_{e} / T\right)}{\exp \left(-\mu_{0} / T\right)+1}, \quad n_{0}^{e}=\frac{m T}{\left(2 \pi \hbar^{2}\right)}, \\
T \geqslant \hbar \omega_{c}, \quad T_{e}=\frac{e E_{\perp} \Delta}{2 \sqrt{2} \ln ^{1 / 2}(\sqrt{R \Delta} / \eta)},
\end{gathered}
$$




$$
V_{a} \geqslant-\frac{\Lambda}{d_{a}} \quad \text { with } \quad \Lambda=\frac{e^{2}\left(\epsilon_{d}-1\right)}{4\left(\epsilon_{d}+1\right)} .
$$

$\epsilon_{d}$ is the dielectric constant of the solid substrate and $d_{a}$ is defined in Eq. (22). $n_{0}^{e}$ is the free electron density of states, and the factor $\exp \left(T_{e} / T\right)$ in the definition of $n_{e}$ reflects the average energy difference between local and free states in the presence of $E_{\perp} \neq 0$. So putting definitions (24) and (25) in Eq. (23), we can derive $\mu_{0}$ versus $V_{a}, T, n_{a}$, and $n_{s}$.

In some limiting cases we get for $\mu_{0}$, from Eq. (24),

$$
\mu_{0} \rightarrow\left\{\begin{array}{l}
-T \ln \left[\left(n_{0}^{e}-n_{s}\right) / n_{s}\right], \quad n_{a} \rightarrow 0, \\
V_{a}-T \ln \left[\left(n_{a}-n_{s}\right) / n_{s}\right], \quad n_{a}>n_{s} .
\end{array}\right.
$$

One can see that a $\theta$-like behavior of the chemical potential, with the amplitude of the jump dependent on $V_{a}$, has developed, see Ref. 19, around

$$
n_{a}=n_{s} .
$$

A more detailed form of $\mu_{0}$ is

$$
\begin{aligned}
2 \epsilon n_{s} x= & {\left[\epsilon\left(n_{0}^{e}-n_{s}\right)+\left(n_{a}-n_{s}\right)\right] } \\
& +\sqrt{\left[\epsilon\left(n_{0}^{e}-n_{s}\right)+\left(n_{a}-n_{s}\right)\right]^{2}+4 \epsilon n_{s}\left(n_{0}^{e}+n_{a}-n_{s}\right)},
\end{aligned}
$$

where

$$
x=\exp \left(-\frac{\mu_{0}}{T}\right), \quad \epsilon=\exp \left(\frac{V_{a}}{T}\right), \quad V_{a}<0 .
$$

The second term in Eq. (28) is always positive. The first term, however, changes sign (in the limit $\epsilon \rightarrow 0$ ) when $n_{a}$ crosses the value $n_{s}$. Before this point there is a strong compensation between the two terms. Such a compensation corresponds to the first asymptote in the definition of $\mu_{0}$ (26). Just after condition (27) the compensation stops, and we have the second asymptote in the definition of $\mu_{0}$ (26).

From Eqs. (24) and (26) we can write the useful asymptote of $n_{l}$ in the limit $n_{l} \ll n_{a}$

$$
n_{l} \simeq \frac{n_{a}}{\left(n_{0}^{e} / n_{s}\right) \exp \left[\left(V_{a}+T_{e}\right) / T\right]+1}
$$

with

$$
n_{0}^{e} \gg n_{s} \quad \text { and } \quad V_{a}<0 .
$$

The situation $n_{l} \ll n_{a}$ is possible, if

$$
\frac{n_{0}^{e}}{n_{s}} \exp \left[\frac{V_{a}+T_{e}}{T}\right]>1 \text {. }
$$

\section{TWO-FRACTION ELECTRON SYSTEM IN THE AC REGIME}

The two-fraction structure of a 2DES is important both in the $\mathrm{dc}$ and the ac regime. In the first case the fraction $n_{l}$ is practically immobile and the conductivity $\sigma_{x x}$ can be described (in the Drude approximation) as ${ }^{19}$

$$
\sigma_{x x}^{\mathrm{dc}}=n_{e} \frac{e^{2} \tau}{m} .
$$

The thickness dependence of $\sigma_{x x}^{\mathrm{dc}}$ follows both from the parameters $\tau(d)$ and $n_{e}(d)$.

In the ac regime, however, the local fraction $n_{l}$ is more "active" because the localization does not stop the dynamics of the $n_{l}$ electrons. To introduce the "top" eigenfrequencies $\omega_{a}$, we use the coupling electron energy in the form

$$
V_{a} \simeq-\frac{\Lambda}{d_{a}(x)},
$$

where

$$
d_{a}(x) \simeq d_{a}(0)\left(1+\frac{\delta^{\prime \prime} x^{2}}{2 d_{a}(0)}\right)
$$

and an expansion of this expression near the minima

$$
V_{a}(x) \simeq-\frac{\Lambda}{d_{a}(0)}+\frac{k_{a} x^{2}}{2},
$$

where

$$
k_{a}=\frac{\Lambda \delta^{\prime \prime}}{d_{a}(0)^{2}} \quad \text { and } \quad \omega_{a}^{2}=\frac{k_{a}}{m} .
$$

In the definitions (30) and (31) we assume that the helium surface is practically flat $\left(R^{2} \gg \Delta^{2}\right)$ and so the local distance $d(x)$ between electron and top profile is only sensitive to $\delta^{\prime \prime}$.

To describe the properties of $\delta^{\prime \prime}$ we have to use the same definition as in Eqs. (20) and (22)

$$
\left\langle\delta^{\prime \prime} \delta^{\prime \prime}\right\rangle=\beta^{2}=\frac{3 \Delta^{2}}{\eta^{4}} .
$$

The corresponding Gaussian distribution $\delta^{\prime \prime}$ of the curvatures is

$$
D(\gamma)=\frac{1}{\left(2 \pi \beta^{2}\right)^{1 / 2}} \exp \left(-\frac{\gamma^{2}}{2 \beta^{2}}\right), \quad \gamma=\delta^{\prime \prime} .
$$

All $\omega_{a}$ modes (31) are split in a magnetic field $\omega_{a}$ $\rightarrow \omega_{a}^{ \pm}$. The existence of this splitting is included in the calculation of dissipation in the low frequency limit $\omega_{a}^{ \pm} \leqslant \omega$, where $\omega$ is the external frequency. For the eigenmodes $\omega_{a}$ $>\omega$ the possible soft contribution of these modes in the general dissipation (50) is neglected due to numerical reasons (such a statement has been formulated in Ref. 20) and the possibility to simplify the algorithm of the fit (see below).

We now make some remarks regarding the well known dimple effect in the ac-free electron behavior: On one hand, there is an experimental indication ${ }^{21}$ with respect to the CRdimple shift on the bulk helium surface. There is also the "dimple" interpretation of a quasi-dc-electron mobility on a thin helium film. ${ }^{22}$ On the other hand, theoretical estimations ${ }^{4}$ show a small probability for the existence of the single electron dimple under the typical helium conditions. Precise dimple effect measurements ${ }^{23}$ within the crystalliza- 
tion problem show a complete correlation between the existence of dimples and the Coulomb crystallization in the 2DES. Our search for the dimple effect in the CR data for electrons on thin helium films on Hostaphan ${ }^{13}$ lead to a negative answer. The same negative result can be extracted from the CR measurements in Ref. 24 for electrons on a helium film with solid hydrogen as a substrate. And finally there is no indication of a CR-dimple shift in the most recent CR measurements ${ }^{25}$ with electrons on bulk helium.

So the conclusion with respect to the CR-dimple shift is mainly negative. To understand the positive conclusions ${ }^{21,22}$ we believe that the data of Ref. 22 can be explained by an alternative scenario, see Ref. 4, where a sharp decrease of mobility versus helium film thickness without a self-trapping effect is proposed. The same motivation is applicable to the interpretation of Ref. 26 for the data of Ref. 27. The data of Ref. 21 partly reflect the influence of the Coulomb crystallization. Besides, also the contribution of the resonator effect (see below) is possible.

\section{CR-MEASUREMENTS IN RESONATORS}

CR for electrons on helium is typically investigated by using a resonator. In this case the conventional way to fix the resonance is reduced to measurements and calculations of the resonator reflection (transmission) versus electron density, magnetic field, helium film thickness, etc. The experiments usually follow these ways. For the calculations, however, the existing ways are quite limited. One uses either the transmission line model ${ }^{21}$ or the simple possibility following from the classical (quantum) motion equations without any indication how the resonator background becomes important. ${ }^{25,28,29}$

The dissipation of the free electrons $Q_{e}^{-1}$ is defined as (see, e.g., Ref. 28)

$$
\begin{gathered}
Q_{e}^{-1}=\operatorname{Re} E_{\| j_{x}} j^{*}\left(\omega, \omega_{c}\right), \\
j_{x}\left(\omega, \omega_{c}\right)=\left(\sigma_{x x}^{\prime}+i \sigma_{x x}^{\prime \prime}\right) E_{\|} \\
\sigma_{x x}^{\prime}=\frac{n_{s} e^{2} \tau}{m} \frac{\left(1+\omega^{2} \tau^{2}+\omega_{c}^{2} \tau^{2}\right)}{\left(1-\omega^{2} \tau^{2}+\omega_{c}^{2} \tau^{2}\right)^{2}+4 \omega^{2} \tau^{2}} \\
\sigma_{x x}^{\prime \prime}=-(\omega \tau) \frac{n_{s} e^{2} \tau}{m} \frac{\left(1+\omega^{2} \tau^{2}-\omega_{c}^{2} \tau^{2}\right)}{\left(1-\omega^{2} \tau^{2}+\omega_{c}^{2} \tau^{2}\right)^{2}+4 \omega^{2} \tau^{2}} .
\end{gathered}
$$

Here $E_{\|}$is the effective electric field along the helium film, $n_{s}$ is the electron density, $\tau$ is the elastic time relaxation, $\omega$ the external frequency, and $\omega_{c}$ the cyclotron frequency.

In reality the electron motion in a resonator is not free. There is a coupling between the electron motion and the resonator mode. The level of this coupling is the essential characteristic of the system $2 \mathrm{D}$ electrons in a resonator.

To estimate such a coupling we have to solve the corresponding eigenproblem. In the case of the resonator in Fig. 3 the lowest eigenmode is (see details in Ref. 30)

$$
\begin{gathered}
\cot (k h)\{\cos [d(q-k)]-i \sigma \tan (k d)\} \\
-\sin [d(q-k)]+i \sigma=0,
\end{gathered}
$$

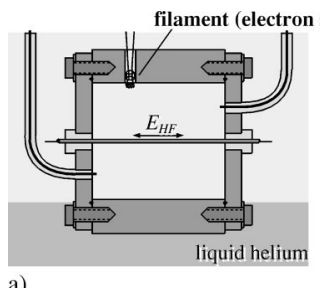

a)

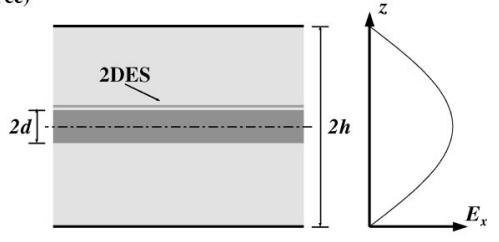

b)
FIG. 3. a) Experimental setup, (b) schematical sketch of the cavity with the dielectric substrate of thickness $2 d$ in the center. On the right a typical profile of the electric field $E$ is shown.

$$
k^{2}=\frac{\omega^{2}}{c^{2}}, \quad q^{2}=\epsilon_{d} \frac{\omega^{2}}{c^{2}}, \quad \sigma=\frac{4 \pi}{c} \sigma_{x x} \cos (k d) \sin (q d) .
$$

The substrate has the thickness $2 d$, the size of the empty resonator is $2 h$, and $\sigma_{x x}$ is from Eq. (34).

From Eq. (38) the role of the coupling constant is shown by the combination

$$
\sigma_{0}=\frac{4 \pi \sigma_{x x}^{0}}{c}, \quad \sigma_{x x}^{0}=\frac{n_{s} e^{2} \tau}{m} .
$$

The critical scale for this parameter is $\sigma_{0} \approx 1$. This is suitable for electrons on bulk helium under the condition: $n_{s}$ $\geqslant 10^{8} \mathrm{~cm}^{-2}$ and $\tau \leqslant 10^{-7} \mathrm{~s}$ (these conditions are presented in Refs. 21 and 29). In this case $\sigma_{0} \leqslant 1$. However, for thin helium films with the electron density from above and typical values $\tau \leqslant 10^{-10} \mathrm{~s}, \sigma_{0} \ll 1$ and so the resonator effect is not important.

Under the conditions $\sigma_{0} \ll 1$ we can therefore use the conventional perturbation theory. Indeed in this case

$$
k=k_{0}+\delta k, \quad \delta k=\delta k^{\prime}+i \delta k^{\prime \prime}, \quad \delta k \ll k_{0},
$$

where $k_{0}$ corresponds to the cavity eigenmode without electrons

$$
\cot \left(k_{0} h\right)-\tan \left[d\left(q_{0}-k_{0}\right)\right]=0 .
$$

If in addition $d \ll h$ then Eqs. (38), (40), and (41) are reduced to

$$
\begin{aligned}
& h \delta k^{\prime \prime}=-\frac{4 \pi}{c} \cos \left(k_{0} d\right) \sin \left(q_{0} d\right) \sigma_{x x}^{\prime}\left(\omega_{0} \omega_{c}\right), \\
& h \delta k^{\prime}=-\frac{4 \pi}{c} \cos \left(k_{0} d\right) \sin \left(q_{0} d\right) \sigma_{x x}^{\prime \prime}\left(\omega_{0} \omega_{c}\right) .
\end{aligned}
$$

The structure (42) of the damping of the eigenmode is the same as the absorption $Q_{e}^{-1}(34),(36)$. Therefore definition (34) is reasonable for a 2 DES in a resonator, if $\sigma_{0} \ll 1$. 


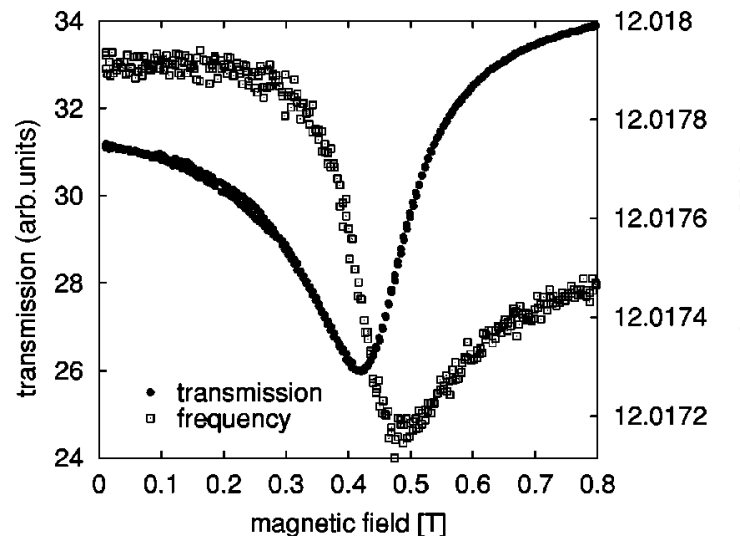

FIG. 4. The transmitted signal (left $y$ axis) and the resonance frequency (right $y$ axis) as function of the magnetic field are shown. The minimum in the transmission, around $0.4 \mathrm{~T}$, determines the cyclotron resonance $\omega_{c}$. The change in frequency, around $\omega_{c}$, gives the evidence of the perturbation of the eigenmode (for further explanation, see text).

In addition to Eqs. (38)-(43) predict the perturbation of the eigenmode (43). This perturbation is shown by our experimental data (see Fig. 4) and also corresponds to the situation $\sigma_{0} \ll 1$.

Now we go back to the two-fraction problem. After discussion of Eqs. (34)-(43) we can present the free electron absorption in the two-fraction model as

$$
\begin{gathered}
Q_{e}^{-1} \propto n_{e} p\left(\omega_{0}, \tau, \omega_{c}\right), \\
p\left(\omega_{0}, \tau, \omega_{c}\right)=\frac{1+\omega_{0}^{2} \tau^{2}+\omega_{c}^{2} \tau^{2}}{\left(1-\omega_{0}^{2} \tau^{2}+\omega_{c}^{2} \tau^{2}\right)^{2}+4 \omega_{0}^{2} \tau^{2}},
\end{gathered}
$$

where $\omega_{0}$ is the frequency from Eq. (41), and $n_{e}$ is the free electron fraction from Eq. (24).

The corresponding absorption $Q_{l}^{-1}\left(\omega_{0}, \omega_{c}\right)$ due to local states is

$$
Q_{l}^{-1}\left(\omega_{0}, \omega_{c}\right) \propto n_{l} \int_{0}^{\infty} D(\gamma) f\left(\omega_{0}^{2} \tau^{2}, \omega_{c}^{2} \tau^{2}, \omega_{a}^{2} \tau^{2}\right) d \omega_{a}
$$

and

$$
f(z, x, t)=\frac{(z-t)^{2}-z-z x}{\left[(z-t)^{2}-z-z x\right]^{2}+4 z(z-t)^{2}}
$$

with

$$
z=\omega_{0}^{2} \tau^{2}, \quad x=\omega_{c}^{2} \tau^{2}, \quad t=\omega_{a}^{2} \tau^{2}
$$

where $n_{l}$ is from Eq. (24), the function $\omega_{a}(\gamma)$ from Eqs. (31), (33), and $D(\gamma)$ from Eq. (33). The total absorption

$$
Q^{-1}=Q_{e}^{-1}+Q_{l}^{-1}
$$

contains six external parameters $d, n_{s}, \tau, \tau_{a}, \Delta$, and $\eta$. It is reasonable to assume that $d$ and $n_{s}$ are well defined independently. The scale of $\tau$ can be estimated using known mobility calculations for 2D electrons on helium. As a result the above calculations have two fit parameters $\Delta$ and $\eta$ which have to be extracted from the experimental data.

In reality this program is too complicated (especially with the introduction of $\tau_{a}$ ). So some simplifications are necessary. First we cut the integration in Eq. (46) by the value $\omega_{0}$ (the reasons for such a simplification have been discussed earlier) and assume that within the interval between 0 and $\omega_{0}$ all $\omega_{a}$ are equally probable. Due to the probability distribution of all $\omega_{a}$ that is true if

$$
\left\langle\omega_{a}^{2}\right\rangle \geqslant \omega_{0}^{2}
$$

The additional assumption

$$
\tau_{a} \approx \tau
$$

cannot be directly proven. But when taking Eq. (49), we can calculate the integral in Eq. (46) in explicit form. In addition the fit shown below demonstrates that for the absorption data condition (49) is valid.

After the simplifications given above the integral in Eq. (46) is transformed into the form

$$
Q_{l}^{-1} \propto n_{l} q\left(\omega_{0}, \tau, \omega_{c}\right),
$$

$q\left(\omega_{0}, \tau, \omega_{c}\right)$

$$
=\frac{\arctan \frac{\sqrt{z}}{1+x+\sqrt{x z}}+\arctan \frac{\sqrt{z}}{(1+x) \sqrt{z}-z \sqrt{x}}+c(z, x)}{2 \sqrt{z}}
$$

and

$$
c(z, x)=\frac{\pi}{2}\{1-\operatorname{sgn}[(1+x) \sqrt{z}-z \sqrt{x}]\} .
$$

The function $c(z, x)$ is only used to switch to another branch of the $\arctan ()$ function. The total absorption has now two fit parameters $\tau$ and $n_{e} / n_{s}$. To extract these numbers from the experimental data it is convenient to fit the combination

$$
\frac{Q^{-1}\left(\omega_{c}^{(\max )}\right)}{Q^{-1}\left(\omega_{c}=0\right)}=\frac{\nu_{e} p\left(\omega_{0}, \tau, \omega_{c}^{(\max )}\right)+\nu_{l} q\left(\omega_{0}, \tau, \omega_{c}^{(\max )}\right)}{\nu_{e} p\left(\omega_{0}, \tau, 0\right)+\nu_{l} q\left(\omega_{0}, \tau, 0\right)},
$$

where

$$
\nu_{e}=\frac{n_{e}}{n_{s}}, \quad \nu_{l}=\frac{n_{l}}{n_{s}}, \quad \text { and } \quad \nu_{e}+\nu_{l}=1
$$

together with the definition of $\omega_{c}^{(\max )}$.

$$
\left.\frac{\delta Q^{-1}}{\delta \omega_{c}}\right|_{\max }=0
$$

Under these conditions there is no guarantee for a good enough reproduction of the absorption line shape. Nevertheless the fit shows a reasonable solution of this problem, see Fig. 5. 

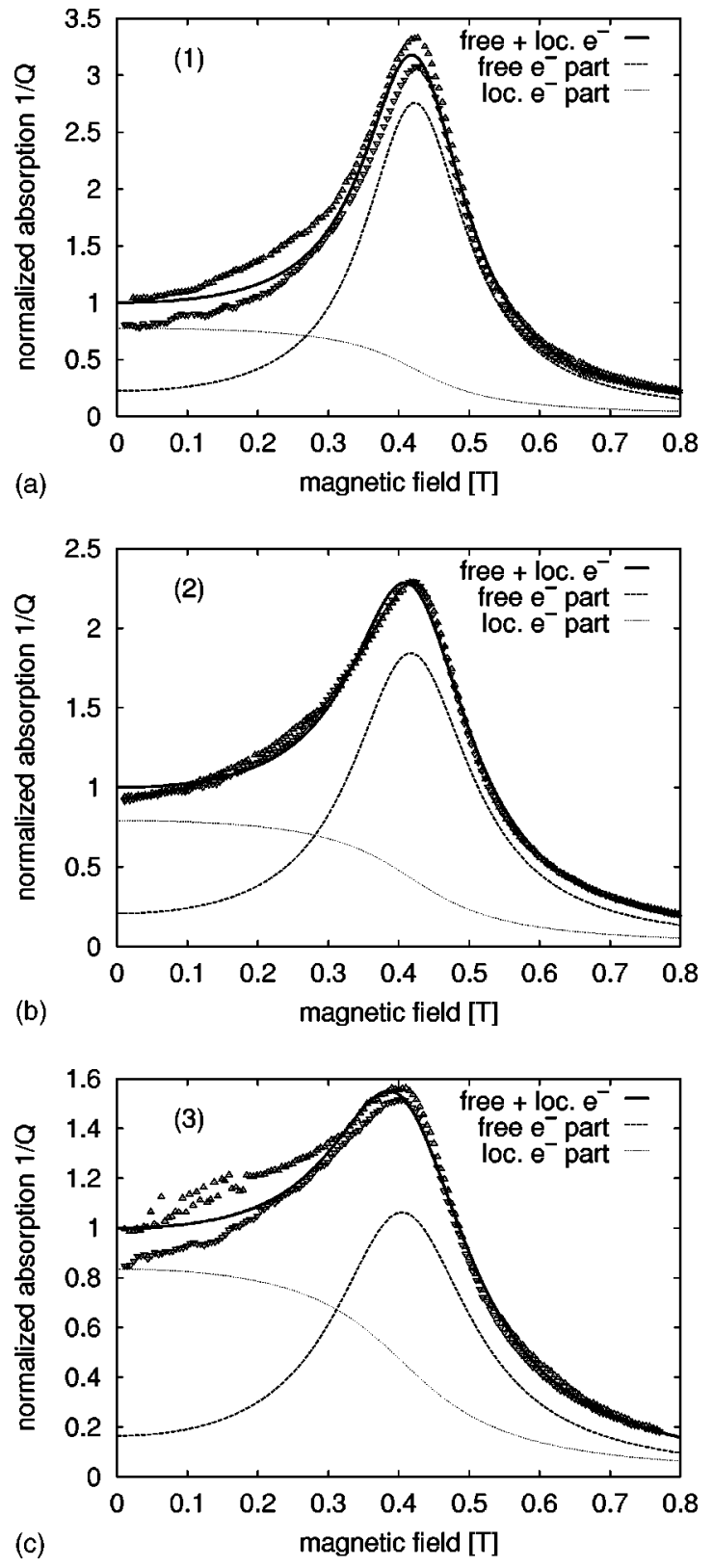

FIG. 5. Shown is the absorption $Q^{-1}$ as function of magnetic field for up $(\triangle)$ and down $(\nabla)$ sweeps. The dashed and dotted lines represent the free and localized electron fraction, the full line is the sum of both fitted to the data. From (1)-(3) the helium film thickness decreases. In (1) $n_{e} \approx 67 \%$, in (2) $n_{e} \approx 60 \%$, and in (3) $n_{e} \approx 49 \%$. These three data sets correspond to the same labeled data points in Figs. 6 and 7.

\section{FITTING THE EXPERIMENTAL DATA}

To investigate the influence of the roughness of the underlying substrate on the CR absorption we have set the helium film thickness by adjusting the bulk helium level below the Si substrate. So varying the distance $h$ from small to large values changes the thickness of the helium film $d$ from thick to thin values. The typical $Q^{-1}$ lines for the different film thicknesses are shown in Fig. 5. It is evident that the CR quality decreases and the asymmetry of the absorption line increases as the helium film gets thinner.
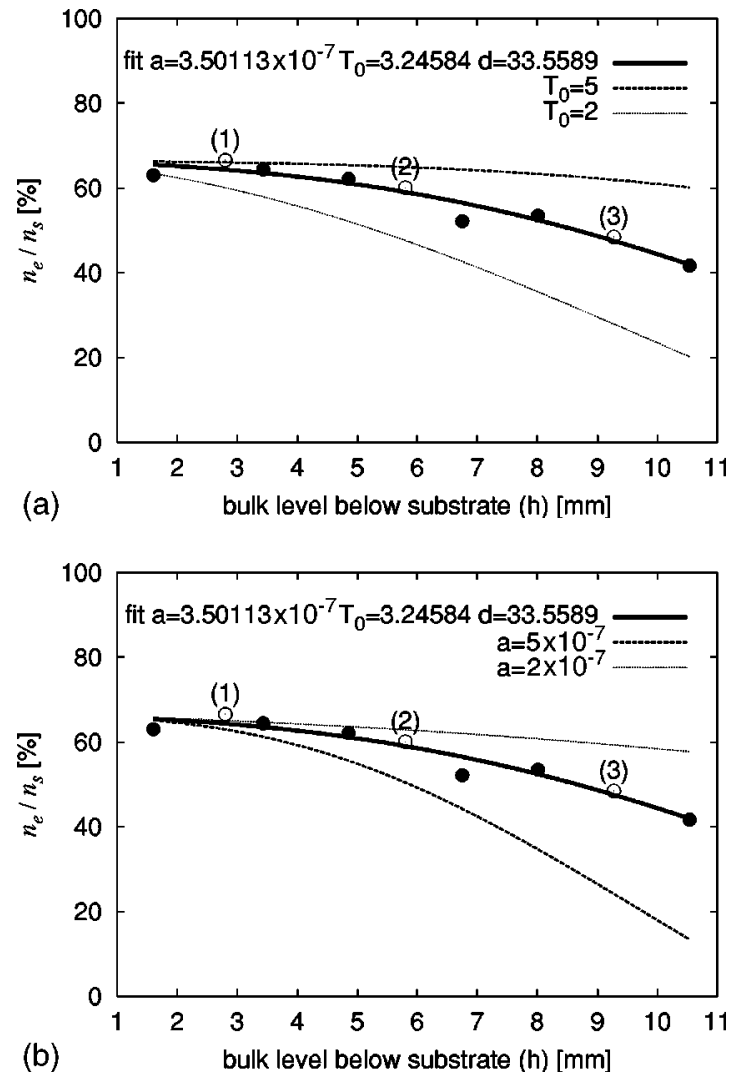

FIG. 6. Dependence of free electron fraction $n_{e}$ as function of distance of bulk helium level below the substrate $h$. (O) and ( $\bigcirc)$ are from fitting to all measured data. Both in (a) and (b) the solid lines present the best fit with the same parameters of $a$ and $T_{0}$. The dashed and dotted lines in (a) show fits with different $T_{0}$ but keeping $a$ fixed, and in (b) with fixed $T_{0}$ but varying $a$. This shows the good agreement with one set of parameters to describe the measured data. The $(\bigcirc)$, labeled (1) to (3), correspond to the same data points as shown in Figs. 5 and 7.

The fit procedure can be split into two parts: first we fit the shape of the $Q^{-1}$ data using Eqs. (44), (47), (50), (52), (54). The details of this fitting are presented in Fig. 5. One can see a quite good reproduction of the features of $Q^{-1}$ in a wide range of the helium film thicknesses. In addition, one can extract information about the electron fractions and the corresponding time relaxation as function of the helium film thickness, see Figs. 6 and 7. The data are, at least qualitatively, understandable: $n_{l}$ monotonically grows and $\omega \tau$ goes down.

Secondly we try to explain the data shown in Fig. 6 and 7. For the progress in the interpretation of $\omega \tau$, our initial description is too crude [see, e.g., the simplification (49)]. Therefore we cannot explain the details of the behavior of $\omega \tau$, Fig. 7. However, within the framework of the fractional structure of the 2DES this problem can be, at least qualitatively, described.

We start from the simplification (48), which looks reasonable considering the excellent fit in Fig. 5, using Eqs. (28), (44), (50). In explicit form the inequality (48) is reduced to the estimation of $\left\langle\delta^{\prime \prime} \delta^{\prime \prime}\right\rangle$. Using definition $\omega_{a}^{2}$ (31) with $d_{a} \leqslant 10^{-6} \mathrm{~cm}, \epsilon_{d} \approx 10$, and the experimental value $\omega$ 


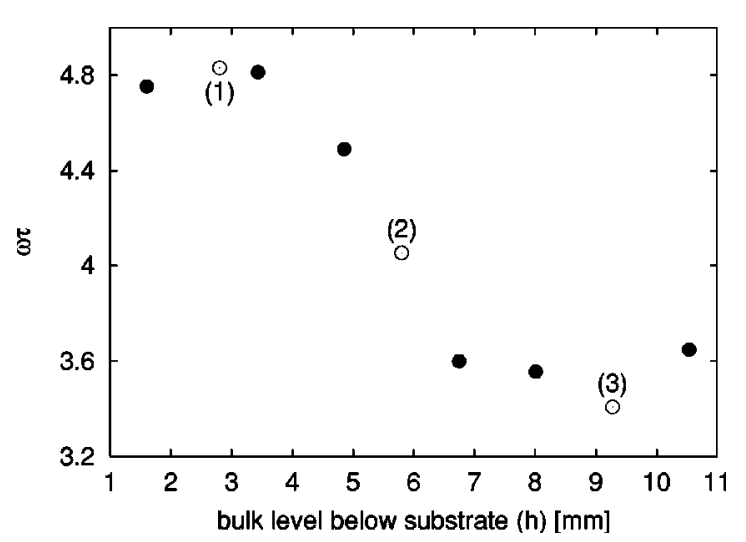

FIG. 7. Shown is the dependence of $\omega \tau$ on the level of the bulk helium below the substrate surface, $h$. The $(\bigcirc)$, labeled (1) to (3), correspond to the same data points as shown in Figs. 5 and 6.

$\cong 2 \pi 10^{10} \mathrm{~s}^{-1}$ we estimate from $\left\langle\omega_{a}\right\rangle \geqslant \omega$ the value

$$
\left\langle\delta^{\prime \prime} \delta^{\prime \prime}\right\rangle=3 \frac{\Delta^{2}}{\eta^{4}} \geqslant 10^{13} \mathrm{~cm}^{-2} .
$$

This estimation shows that a smooth approximation (25) for $V_{a}$ is not realistic enough. It has to be reduced, using, e.g., the potential energy between the pointlike charge and the spherical dielectric instead of the semi-infinite dielectric. Unfortunately the spherical image problem has no analytic solution, see Ref. 31. To obtain an analogy to the problem of the cylindrical image potential, we use for $V_{a}$ the expression

$$
V_{a} \rightarrow-\frac{2 \Delta}{\left(d_{a}+a\right)} \frac{a^{2}}{\left[\left(d_{a}+a\right)^{2}-a^{2}\right]},
$$

where

$$
a^{2}=\left\langle\delta^{\prime \prime} \delta^{\prime \prime}\right\rangle^{-1} .
$$

In the limit $a \gg d_{a}$ expression (56) is reduced to $V_{a}$ from Eq. (25).

If so, we go to the definition of $n_{e}$ (24) with $n_{a}$ from Eq. (18), $E_{\perp}=2 \pi e n_{s}$ and $V_{a}$ from Eq. (56). Using $T_{e}$ from Eq. (24) and $a$ from Eq. (56) as a fit parameter we can explain the data in Fig. 6 with

$$
T_{e} \cong 3.19 \mathrm{~K} \text { and } a \cong 2.25 \times 10^{-7} \mathrm{~cm} .
$$

The fitting lines, Fig. 6 , are very sensitive to variations both in $T_{e}$ [Fig. 6(a)] and $a$ [Fig. 6(b)]. Therefore the level of accuracy for the above numbers is not realistic, but the scale of these numbers is reliable.

It is necessary to note that the scenario presented in Fig. 6 has some artificial correction. The "beginning" of all these lines corresponds to the free electron behavior of the 2DES when regime $n_{l} \ll n_{a}$ takes place. But in this case the "plateau" (i.e., the first three data points from the left side of Fig. 7) should be around $100 \%$ instead of the measured $70 \%$. To explain such a shift, which is not sensitive to the thickness of the helium film, we have to remember the geometry of our setup. The silicon substrate has a finite area with a sharp perimeter. The equilibrium helium film around this perim- eter, just after crossing the point $h=0$, is inevitable thinner than on the main silicon area. And this should cause localization of the edge electrons while the electrons above the substrate are still free. This, we expect, causes a sharp drop in the free electron fraction which may well be around $30 \%$. Exact measurements in this range, however, still have to be done.

Finally from the above numbers for $T_{e}$ and $a$, using definitions (56), (32), and (24), and $n_{s} \cong 10^{9} \mathrm{~cm}^{-2}$ we get

$$
\begin{gathered}
\Delta \cong \frac{2 T_{e}}{\pi e^{2} n_{s}} \cong 8 \mathrm{~nm}, \\
\eta^{4}=3 \Delta^{2} a^{2} \cong(6 \mathrm{~nm})^{4} .
\end{gathered}
$$

These values, being in the nm regime, are reasonable and typical for a real surface.

\section{CONCLUSIONS}

The consideration above is related to a quite general problem of the behavior of a 2DES on a liquid helium film in the presence of a substrate with the usual surface roughness. The use of a thin helium film is desirable to increase the values of the critical electron density. However, simultaneously the influence of the surface roughness grows and becomes an important factor. The interaction of the 2DES with the substrate roughness is not direct. Rather it is modified by the nonuniform profile of the helium film. Such a "screening" effect is especially strong if the roughness amplitude $\delta(x)$ exceeds the equilibrium helium film thickness $d: \delta(x) \gg d$. Under these conditions the conventional perturbation theory does not work, and the question arises, how to describe such a quite typical situation.

We here propose the two-fraction model to be suitable to describe the behavior of a 2DES when $\delta(x) \gg d$. We have defined the density of the active tops $n_{a}$, which is dependent on the characteristics of the random rough substrate. The definition of the localized $n_{l}$ and free electron fraction $n_{e}$ as a function of $n_{a}, n_{s}, T$, and $d$, as well as the description of the dynamics of the localized electrons is given. All this information has been used to interpret the CR data for $2 \mathrm{D}$ electrons on thin helium films. The most prominent feature of these data is the unusual asymmetry of the absorption line shape and its growing as the helium film thickness decreases. The two-fraction picture explains this asymmetry quite well, see Fig. 5. The developed fit program helps to extract important information about $n_{l}$ and $n_{e}$ from the experimental data. In addition, we can explain, at least qualitatively, the behavior of $n_{e}(h)$, see Fig. 6.

In the scenario presented here several approximations and assumptions are made: we take the substrate roughness to be Gaussian-like and one dimensional, we use the simple acOhm's law instead of the self-consistent resonator response, we neglect a possible distribution in the time relaxation $\tau_{a}$, propose a quite naive modification (56) of the potential $V_{a}$, and introduce the influence of possible localized states along the perimeter of the substrate. Within these approximations 
the obtained quantitative information might be not very reliable. However, the qualitative conclusions, i.e., (a) the twofraction model has a field of application, (b) the CR asymmetry has its origin in the two-fraction scenario, and (c) there is an overlap between the dc- and ac- two-fraction predictions, is very reasonable.

\section{ACKNOWLEDGMENTS}

This activity is supported partly by the Deutsche Forschungsgemeinschaft, Forschergruppe "Quantengase," the INTAS Network 97-1643, and the RFBI Grant No. 0202 17082 .
${ }^{1}$ V. Shikin and Yu. Monarkha, 2D Charged Systems in Liquid Helium (Nauka, Moscow, 1989).

${ }^{2}$ S.A. Jackson and P.M. Platzman, Phys. Rev. B 24, 499 (1981); 25, 4886 (1982).

${ }^{3}$ O. Hipolito, G.A. Farias, and N. Studart, Surf. Sci. 113, 394 (1982).

${ }^{4}$ F.M. Peeters and S.A. Jackson, Phys. Rev. B 34, 1539 (1986).

${ }^{5}$ D. Chernikova, Sov. Phys. JETP 6, 737 (1980).

${ }^{6}$ V. Tatarskii, Sov. Phys. JETP 12, 255 (1986).

${ }^{7}$ H. Etz, W. Gombert, W. Idstein, and P. Leiderer, Phys. Rev. Lett. 53, 2567 (1984).

${ }^{8}$ A.P. Volodin and V.S. Edel'man, JETP Lett. 37, 7 (1983).

${ }^{9}$ M. Saitoh, Phys. Rev. B 40, 810 (1989).

${ }^{10}$ H.W. Jiang and A.J. Dahm, Surf. Sci. 228, 352 (1990).

${ }^{11}$ M.A. Paalanen and Y. Iye, Phys. Rev. Lett. 55, 1761 (1985).

${ }^{12}$ D. Cieslikowski, A.J. Dahm, and P. Leiderer, Phys. Rev. Lett. 58, 1751 (1987).

${ }^{13}$ J. Klier, T. Günzler, A. Würl, P. Leiderer, G. Mistura, E. Teske, P. Wyder, and V. Shikin, J. Low Temp. Phys. 122, 451 (2001).

${ }^{14}$ E. Cheng and M.W. Cole, Phys. Rev. B 38, 987 (1988).

${ }^{15}$ A. Valkering, J. Klier, and P. Leiderer, Physica B 284, 172 (2000).

${ }^{16}$ Yu.P. Monarkha, Sov. Phys. JETP 18, 145 (1992).

${ }^{17}$ V. Tikhonov, Usp. Fiz. Nauk 77, 449 (1962).

${ }^{18}$ J. Blakemore, Semiconductor Statistic (Pergamon Press, Oxford, 1962).
${ }^{19}$ V. Shikin, J. Klier, I. Doicescu, A. Würl, and P. Leiderer, Phys. Rev. B 64, 073401 (2001).

${ }^{20}$ B.A. Wilson, S.J. Allen, and D.C. Tsui, Phys. Rev. B 24, 5887 (1981).

${ }^{21}$ V. Edel'man, Sov. Phys. JETP 50, 338 (1979).

${ }^{22}$ O. Tress, Y.P. Monarkha, F.C. Penning, H. Bluyssen, and P. Wyder, Phys. Rev. Lett. 77, 2511 (1996).

${ }^{23}$ F. Gallet, G. Deville, A. Valdes, and F.I.B. Williams, Phys. Rev. Lett. 49, 212 (1982); G. Deville, A. Valdes, E.Y. Andrei, and F.I.B. Williams, ibid. 53, 588 (1984); D.C. Glattli, E.Y. Andrei, and F.I.B. Williams, ibid. 60, 420 (1988).

${ }^{24}$ V.S. Edelman and M.I. Faley, J. Low Temp. Phys. 52, 301 (1983).

${ }^{25}$ F.C. Penning, O. Tress, H. Bluyssen, E. Teske, M. Seck, P. Wyder, and V. Shikin, Phys. Rev. B 61, 4530 (2000).

${ }^{26}$ G.E. Marques and N. Studart, Phys. Rev. B 39, 4133 (1989).

${ }^{27}$ F.F. Mende, Yu.Z. Kovdrya, and Y.A. Nikolaenko, Sov. Phys. JETP 11, 355 (1985).

${ }^{28}$ G. Dresselhaus, A.F. Kip, and C. Kittel, Phys. Rev. 98, 368 (1955).

${ }^{29}$ E. Teske, Y.P. Monarkha, M. Seck, and P. Wyder, Phys. Rev. Lett. 82, 2772 (1999).

${ }^{30}$ A. Würl, J. Klier, P. Leiderer, and V. Shikin, J. Low Temp. Phys. 126, 511 (2002).

${ }^{31}$ L. Landau and E. Lifshitz, Electrodynamics of Continuous Media (Pergamon, Oxford, 1984). 